\title{
CRISPR Gene Therapy in Dentistry
}

\author{
Anjana Goyal $^{1 *}$, Reena Doomra ${ }^{2}$, Aayushi Garg ${ }^{3}$, Kruthiventi Hemalata ${ }^{4}$ \\ ${ }^{1}$ Head, Department of Biochemistry, Manav Rachna Dental College, Faridabad \\ ${ }^{2}$ Head, Department of Pharmacology, Manav Rachna Dental College, Faridabad \\ ${ }^{3}$ BDS $1^{\text {st }}$ Year student, Manav Rachna Dental College, Faridabad \\ ${ }^{4}$ BDS $1^{\text {st }}$ Year student, Manav Rachna Dental College, Faridabad
}

Received: 15-05-2019 / Revised: 18-6-2019 / Accepted: 25-06-2019

\begin{abstract}
CRISPR or Clustered Regulatory Interspaced Short Palindromic Repeats is a family of DNA sequence. They are found in the genomes of prokaryotic organisms like bacteria. CRISPR gene editing is a new method by which genomes of organisms can be edited. It aims to treat many harmful diseases like HIV. Also, CRISPR arrays are used to treat dental plaque biofilms (EPS; extracellular polysaccharide) caused by Streptococcus mutans
\end{abstract}

Keywords: CRISPR, genomes, genes, organisms

\section{Introduction}

In CRISPR gene therapy Cas9 (CRISPR associated protein 9), a protein is used. It can act as a molecular scissors (endonuclease) and can cut DNA and thus can alter genome. Cas9 enzyme forms CRISPR-Cas9 along with CRISPR sequences, which is used to alter genes. Cas9 is obtained from bacteria Streptococcus pyogenes, a gram +ve bacteria. This terminology was adopted from natural defense mechanism of bacteria. Using this technique, gene can be turned off and new genes or their functions can be added or changed.

\section{History}

In 2012, Jennifer Doudna and Emmanulle Charpentier were the first to propose that CRISPR Cas9 could be used for editing of genes. Genomic editing in eukaryotic cells was possible in late 1900's also but it was ineffective to be applied on larger scale. In 2010, synthetic nucleases called transcription activator-like effector nucleases (TALEN's) were used to target double stranded break to specific location on DNA strand. These require creation of custom protein for each targeted DNA sequence, which is time consuming. CRISPR are much easier to make, as only short RNA sequence has to be produced. [1]

*Correspondence

Dr. Anjana Goyal

Head, Department of Biochemistry, Manav Rachna Dental College, Faridabad

E Mail: goyalanjana17@gmail.com

\section{Application}

Cas9 genomic modification has allowed for the quicker and efficient generation of transgenic models. It can be easily introduced into target cells via plasmid in order to model the spread of disease and cell response and defense to ingestion. [2]CRISPR-Cas9 can be used to edit DNA of organisms in vivo and entire chromosomes can be eliminated from any organism. This method is useful for treating chromosomal disorders like aneuploidy or polyploidy which includes disorders like Downs Syndrome, Keinfactor's Syndrome, etc. [3] Human tripronuclear zygotes' gene were effectively edited by CRISPR-Cas9 technique. Chinese scientists P. Liang and Y.Xu first described it. Out of 54 embryos 28 of them were observed to have successful cleavage of Beta-Hemoglobin. Though DNA repair in vivo is much more complicated process than repair derived from stem cells, it is more particular and precise. CRISPR may have application in tissue engineering and regenerative medicine, such as by creating blood vessels of humans that lack expression of protein such as MHC Class II (Major Histocompatibility complex; which are found on B cells which are important in initiating immune response), which often cause transplant rejection to CMI. [4]

\section{CRISPR and Cancer}

Gene editing trials have begun in the USA on two cancer patients. With CRISPR technique researchers aim to modify immune cells so that they become cancer killers. Our immune system has evolved to fight 
off all the invaders (antigens), but in some diseases like cancer, immune system does not work. In order to treat cancer, researchers are using CRISPR and delete two genes in patient $\mathrm{T}$ cells (Immune cells). One of the genes to be removed makes a "checkpoint" molecule PD-1, that cancer cells exploit to put brakes on the immune system. Further edit deletes the receptors that immune cells use to sense danger. Instead an engineered receptor will instead steer them towards particular tumors. This will allow the cell to fight cancer.

\section{CRISPR and dentistry}

CRISPR- Cas9 will be able to identify the causative gene in many oral pathologies and disorders including childhood caries. It will also help in identification of genes that are having tumor-promoting properties of genes that cause cancer. With it, the ability to delete or replace genes, CRISPER can improve diagnosis and treatment of carcinoma of the oral cavity.CRISPRCas9may target viruses and erase them. This may provide a cure for virus disease like Herpes caused by Herpes simplex virus. Caries is mainly caused by Streptococcus mutans, which is a gram +ve bacteria. The harm-causing factor of this bacterium utilizes sucrose to synthesize extracellular polysaccharide (EPS), which leads to the formation of dental plaque biofilm. Using CRISPR technique, self-targeting
CRISPR arrays are designed and cloned to form plasmids. As the genes are edited successfully, high reduction of EPS synthesis has been observed. [5]

\section{Reference}

1. Youngs "Crispr and other genome editing tools Boost Medical Research and Gene Therapy's reach" MIT technology review 2014-04-13

2. DOWLE ."Modelling Disease in vivo with CRISPR/Cas9" Trends in molecular medicine .2015;21(10):609-621

3. ZuoE, Huox, Yaox, Hux, SunY, Yin et al. (2017) CRISPr/Cas9- mediated targeted chromosome elimination genome biology

4. Abrahimi P, Chang WG, Klugerms, Quang Y, Jellides G, Saltzman WM, Paba JS . "Egg; cient gene disruption in cultured primary human endothelial cells by CRISPR/Cas9"2015;3(4):24

5. A. Avilés-Reyes, I. A. Freires, J. K. Kajsafasz, D. Barbieri, J.H. Miller, J. A. Lemos, J. Abranches. Molecular Oral Microbiology. "Whole genome sequence and phenotypic characterization of a Cbm+ serotype e strain of Streptococcus mutans $^{\text {" }} .2018 ; 33(3): 23$

\section{Conflict of Interest: None Source of Support: Nil}

